\title{
Introducing Rubrics as an Objective Assessment Strategy
}

\author{
Bazirete, Oliva ${ }^{1}$, Ruhumuliza, Cairn ${ }^{1}$ \\ ${ }^{1}$ University of Rwanda, College of Medicine and Health Sciences, Rwanda
}

\section{Background}

Clinical assessment of student performance is consistently identified as a challenge, even for experienced faculty. It is, by its nature, somewhat subjective and may not reflect student performance accurately. The Midwifery department at the School of Nursing and Midwifery (SONM), within the College of Medicine and Health Sciences (CMHS) at the University of Rwanda (UR), has taken on the challenge of trialing innovative teaching and assessment methodologies, especially with the baccalaureate completion cohorts. Performance rubrics have historically been used in secondary and higher education to assess classroom activities but have recently begun to be actively integrated in clinical settings as well.

\section{Description}

Beginning with the second semester of the last academic year, and continuing this year, rubrics have been incorporated as assessment tools within the clinical practice settings for the year one cohort of the undergraudate midwifery program. A Reflective Journal Rubric is being implemented to support formative assessment. The Lasater Clinical Judgment Rubric was revised following faculty feedback and is being used as a summative assessment tool. Additional rubrics are being used for assessing the teaching practicum for the year two cohort of midwifery students to assess their clinical and classroom performance.

\section{Lessons Learned}

While findings related to this innovation are still preliminary, strong positive feedback is reported by both faculty and students regarding the use of the performance rubrics because of their perceived objectivity and accuracy of clinical assessment. Rubrics ease the grading experience for faculty and students. Comments and observations are incorporated into the rubric, which shortens the grading time and increases the quality of assessment. Feedback from faculty and students are very valuable in refining the rubrics to fit the local context more precisely.

\section{Conclusions}

Performance rubrics are developed reflecting the classroom and clinical outcomes, hence aligning clearly with the curriculum. Students and lectures are benefiting greatly from the use of performance rubrics as a way of enhancing critical thinking and clinical performance. Rubrics may be effective in a variety of classroom and clinical settings.

Key words: rubrics, performance, assessment 